\title{
Afucosylated biosimilars need afucosylation specific
} assays

\begin{abstract}
Follow-on biologics or biosimilars face formidable development challenges when faced with the need to match glycosylation-related critical quality attributes. This is due in part to the inability to control glycoform heterogeneity during the manufacturing process. Here we discuss critical challenges associated with manufacturing followon afucosylated IgG1s; products that involve $\mathrm{Fc}$ effector function as a part of their mechanism of action. Specifically we discuss the general difficulty associated with the need to match both reference product afucosylation content and biological activity. Finally, we discuss recent experimental and theoretical developments by multiple groups that have opened the door to the development and implementation of robust afucosylation specific assays; powerful tools essential to the development of afucosylated antibody biosimilars.
\end{abstract}

Volume 4 Issue 5 - 2017

\author{
John D Chung,' Peter L Zhan² \\ 'Chung Bioengineering Consulting and Department of \\ Chemistry, Mendocino College, USA \\ ${ }^{2}$ Feinberg School of Medicine, Northwestern University, USA
}

\begin{abstract}
Correspondence: John D Chung, Chung Bioengineering Consulting and Department of Chemistry, Mendocino College, USA, Tel 7073496672, Fax 7072631908,

Email jdchung@alum.mit.edu
\end{abstract}

Received: August 04, 2017 | Published: November 30, 2017

Keywords: antibody, biosimilars, follow-on biologic, afucosylation, activity, antibody, fc glycan, glycosylation, heterogeneity, quality

Abbreviations: CQA, critical quality attributes; MOA, mechanism of action; ADCC, antigen dependent cellular cytotoxicity

\section{Introduction}

Therapeutic antibodies produced by cell culture exhibit considerable glycoform heterogeneity and this heterogeneity may introduces formidable challenges to the development of follow-on biologics or biosimilars. Non-template protein expression is difficult to control since many factors including host cell genetic background and fermentation conditions, all confidential information, significantly affect glycosylation. Since it is generally impossible to precisely control glycosylation, follow-on biologics will always exhibit some degree of variation from the innovators or reference product. The natural question is "What is good enough?" When glycosylation has little or no impact of drug efficacy, differences in glycoform distributions are tolerable since they are therapeutically inconsequential. In such cases, follow-on biologics development proceeds in a straightforward manner. However when glycosylation is known to affect drug efficacy, the responsible glycans become critical quality attributes (CQA) that must be tightly controlled and reproduced during the manufacturing process. Depending on the nature of the CQA, this can introduce formidable challenges to the follow-on manufacturer since they must not only physically reproduce the physical substance but they must also prove that their product is biologically equivalent to the reference product. Since some degree of variation is inevitable, developers must be equip themselves with the scientific metrics that will allow then to prove that their product is "good enough."

These challenges are exemplified by IgG1 drugs whose mechanism of action (MOA) involves Fc mediated antigen dependent cellular cytotoxicity (ADCC). It is now well known that the absence of a core fucose molecule bound to the $\mathrm{Fc}$ region of IgG1 dramatically affects Fc $\gamma$ RIIIa binding and Fc $\gamma$ RIIIa-mediated antigen dependent cellular cytotoxity (ADCC). ${ }^{1,2}$ Afucosylated IgG1 exhibit greatly enhanced Fc $\gamma$ RIIIa binding and increased Fc $\gamma$ RIIIa-mediated antigen dependent cellular cytotoxity (ADCC). These considerations directly impact biologics such as Rituximab and Palivizumab whose MOA involves
Fc mediated effector functions. ${ }^{3,4}$ Therefore follow-on biologics in this class are faced with the task of reproducing the distribution of afucosylated glycoform and demonstrating equivalent biological activity.

\section{Discussion}

Manufacturing antibodies with well-defined and well-characterized afucosylation content to match reference product specifications has proven to be wrought with uncertainties. Matching afucosylation content is a complex task because afucosylation content is a lumped or aggregate variable that is determined by the overall distribution of many different afucosylated glycoforms. Four major classes of afucosylated glycoforms have been designated GO-F, G1-F, G2-F and high mannose along with numerous minor species. It should be noted that the high mannose class is actually comprised of many different glycoform many of which continue challenge detection. Therefore many combinations of these classes of glycoform can lead to the same overall afucosylation content. Further complicated arise from the fact that some afucosylated Fc glycans are undetectable by certain analytical methods so that method dependent considerations can be important. ${ }^{5}$ It is common to match afucosylation content by simply comparing the relative amounts of the major classes of glycoform along with the overall afucosylation content. Since identically matching each species is a practical impossibility, follow-on biologics developers seeks to attain "similar" profiles of the major glycoform while matching the overall afucosylation content.

Much more challenging than matching overall afucosylation content is matching reference product biological activity. Afucosylation content is a physical quantity that is directly measurable so that little ambiguity exists in the interpretation of the data. This is not the case with biological activity. Similar to afucosylation content, biological activity is also a lumped variable that reflects the sum of activities of many different glycoforms, afucosylated and afucosylated, evaluated using a specific set of assay conditions. Since the various glycoform are present in different amounts and many have been shown to exhibit different specific activities, overall activity is a more convoluted 
variable. At high afucosylation content $(\sim 90 \%$ afucosylated $\mathrm{Fc}$ glycans), overall activity principally reflects the activity of the afucosylated antibodies. However at low afucosylated content ( $10 \%$ afucosylated $\mathrm{Fc}$ glycans), a region relevant to many follow-on biologics, fucosylated antibodies make significant contributions to the overall activity so that the interpretation of activity becomes unclear. Activity represents the weighted sum of afucosylation specific and non-specific activities. Because undetectable amounts of afucosylated glycoform exist, it remains unclear whether the apparent activity of afucosylated glycans is real or due to the aggregate activity of numerous undetected afucosylated glycans. The later would result in afucosylated antibody specific activity depending on the amount of "contaminants" present and this has the potential to complicate attempts to match both afucosylation content and sample activity.

The development of follow-on biologics would greatly benefit from the implementation of methods capable of determining afucosylation specific activity. Restricting activity analysis to simply comparing activity of a biosimilar candidate to a reference product fails to address a basic requirement that must be met as the result of classifying "afucosylation content" a CQA. As a CQA, it is not enough for follow-on developers to simply measure afucosylation content. They must also show that the measured afucosylation content is real or biologically active. In other words, they must dissect afucosylation specific from afucosylation non-specific activity. The importance of this dissection cannot be overemphasized because it is well established that afucosylated antibodies exhibit significant levels of activity at low afucosylation content. Without the means to differentiate specific versus non-specific activity, measurements of afucosylation content lack meaning. In this regard it is important to recall that afucosylation content has been designated a CQA because of its enhanced biological activity. Therefore, measurement of afucosylation content must be consistent with measurement of biological activity as both variables are related. Only after this link is established experimentally can activity meaningfully interpreted. Establishing this link will greatly advance the characterization of biosimilars as well as provide a reference in which to assess "goodness of match."

Afucosylation specific assays are currently in existence. The work of a number of groups over the years has been directed at the development of afucosylation specific assays. ${ }^{6-9}$ Using controlled mixtures involving homogeneous afucosylated and fucosylated antibodies to control overall afucosylation content, Chung et al. ${ }^{6}$ first demonstrated the existence of a linear dose-response curve between activity and afucosylation content. This does response curved subsequently allowed them to separate afucosylation specific activity, for the homogeneous afucosylated antibody, from the background activity. Zhan and Chung ${ }^{7}$ later provided a theoretical foundation for this work. More recently, Chung and Zhan ${ }^{8}$ describe detailed algorithms for implementing afucosylation specific assays over a range of experimental conditions. Many of the technical difficulties associated with acquiring the reagents needed to implement afucosylation specific assays at low afucosylation content appear to have been solved by the elegant work of Louie and coworkers. Louie et al. ${ }^{9}$ used a $\mathrm{FX} \mathrm{KO} \mathrm{CHO} \mathrm{cell} \mathrm{line} \mathrm{and} \mathrm{controlled} \mathrm{fucose} \mathrm{feeding} \mathrm{to}$ produce antibodies with different levels of afucosylation. This method allowed them to use a single cell line thus enabling the expression of different levels of afucosylated antibodies without significantly altering the overall glycoform distribution. It is important to highlight that the development and implementation of afucosylation specific activity assays has not been a straightforward task. Complications arise because afucosylation content represents the total amount of afucosylated $\mathrm{Fc}$ glycans with little regard to how these glycans are distributed among antibody molecules. Since each antibody molecule contains two $\mathrm{Fc}$ glycans, or potential afucosylation sites, combinatorial considerations result in three distinct afucosylated antibody forms since an antibody may contain zero, one or two fucose molecules. Since the activity of each moiety has been shown to be different, ${ }^{7}$ both the distribution and the overall afucosylation content determine activity. These complications have resulted in the possibility that samples with similar afucosylation can have very different activity. ${ }^{7}$ Therefore, the means to produce afucosylated antibodies at different levels and a theoretical understanding of the impact of afucosylationbased heterogeneity are needed before reliable and robust assays could be implemented. The works highlighted in this article now provide a straightforward means implement these assays in practice.

\section{Acknowledgements}

None.

\section{Conflict of interest}

The author declares no conflict of interest.

\section{References}

1. Mori K, Kuni-Kamochi Y, Yamane-Ohnuki N, et al. Engineering Chinese hamster ovary cells to maximize effector function of produced antibodies using FUT8 siRNA. Biotechnol Bioeng. 2004;88(7):901-908.

2. Yamane-Ohnuki N, Kinoshita S, Inoue-Urakubo M, et al. Establishment of FUT8 knockout Chinese hamster ovary cell: An ideal host cell line for producing completely defucosylated antibodies with enhanced antibodydependent cellular cytotoxity. Biotechnol Bioeng. 2004;87(5):614-622.

3. Cartron G, Dacheux L, Sallies G, et al. Therapeutic activity of humanized anti-CD20 monoclonalantibody and polymorphism in IgG Fc receptor Fc $\gamma$ RIIIa gene. Blood. 2002;99(3):754-758.

4. Hiatt A, Bohorova N, Bohorova O, et al. Glycan variants of the respiratory Syncytial virus antibody with enhance effector function and in vivo efficacy. Proc Natl Acad Sci USA. 2014;111(16):5992-5997.

5. Du Y, May K, Xu W, et al. Detection and quantitation of afucosylated $\mathrm{N}$-linked oligosaccharides in recombinant monoclonal antibodies using enzymatic digestion and LC-MS. J Am Soc Mass Spectrom. 2012;23(7):1241-1249.

6. Chung S, Quarmby V, Gao X, et al. Quantitative evaluation of fucose reducing effects in humanized antibody on Fc $\gamma$ receptor binding and antibody dependent cell-mediated cytotoxicity activities. MAbs. 2012;4(3):326-340.

7. Zhan PL, Chung JD. hallenges with afucosylation content in antibodybased drugs: Guidance provided by mathematical modeling. Biotechnol Prog. 2015;31(3):775-782.

8. Chung JD, Zhan PL. General solutions to decompose heterogeneous compositions using antibody afucosylation as a model system. Biotechnol Prog. 2017;33(2):500-510.

9. Louie S, Haley B, Marshall B, et al. FX knockout CHO hosts can express desired ratios of afucosylated or afucosylated antibodies with high titers and comparable product quality. Biotechnol Bioeng. 2017;114(3):632644. 